\begin{tabular}{ccc}
\hline International Journal of Engineering \& Technology, $7(2.24)(2018) 109-112$ \\
SPC & Website $:$ www.sciencepubco.com/index.php/IJET \\
Research paper & Technology \\
\hline
\end{tabular}

\title{
Role of Blockchain in the Internet-of-Things (Iot)
}

\author{
Bragadeesh SA ${ }^{1}$, A. Umamakeswari² \\ ${ }^{1}$ Research Scholar, ${ }^{2}$ Associate Dean - CSE, School of Computing. SASTRA Deemed to be University, Thanjavur- 613401. \\ *Corresponding Author Email: sa_bragadeesh@yahoo.co.in
}

\begin{abstract}
The proliferation of Internet of Things (IoT) has brought about a myriad of applications to life which range from smart homes, smart energy, smart buildings, smart cities to almost smart everything. This has been made possible by fully exploiting the services of cloud infrastructure, communication technologies, improved sensing devices and advancements in embedded devices which helps in incorporating intelligence even on tiniest of chips. Blockchain is a decentralised distributed approach that can facilitate management, control and also provide security. The application of blockchain is not restricted to cryptocurrency and security. It is perceived that Blockchain in Convergence with Artificial Intelligence, Cognitive Computing and Mobile Edge Computing can provide numerous prospects for framing solutions for IoT. Any application or system which deploys a IoT network can make use of Blockchain to achieve simplicity, transparency, security and cost efficiency. This paper tries to identify some key areas in which Blockchain can be used to exploit the IoT infrastructure and overcome the challenges faced. Few possible application domains in which blockchain can revolutionize the outcomes have been highlighted. A real-time case study in which Blockchain has been used to provide valuable benefits is discussed.
\end{abstract}

Keywords: Internet of Things, Blockchain.

\section{Introduction}

Information Technology (IT) has scaled new peaks and application domains and solutions which were not even considered possible have been established with near perfect solutions. Internet has played a major role in bringing technology to the end user which has helped people reimagine the world to suit their specific needs. This has been hugely made possible by the advent of IoT. IoT constitutes devices that are interconnected to achieve a specific objective by exchanging data and control information among them. Since the time IoT had come to the fore, it has galvanised the IT revolution and has enabled intelligent solutions to almost every single user's requirements. The vastly improved embedded systems technology has further added to the capabilities to deliver customized solutions across multiple domains. The principal factor which acts as a driving force for all IoT solutions is to derive intelligence from the available data in order to make better decisions, perform analytics, estimate and predict parameters and more often than not perform monitoring and control operations. As with adoption of any new technology, realising the full potential of IoT has not been achieved yet. This is partly due lack of standards, heterogeneous nature of devices, sheer number of devices that needs to be connected, diversified communication protocols, and most important of all security. Security acts as a major bottleneck for adoption of IoT solutions. Apart from security the key challenges in deployment of IoT solutions are

- Technology maturity

- $\quad$ Finding budget

- $\quad$ Time to market

- $\quad$ Lack of skilled workers/experts

- Data quality

- Integration complexity
Over the past few years IoT has been the buzzword across industries, technical experts, academicians, researchers and almost anybody who has access to technology. Recent trends indicate more focus towards Edge Computing, AI (Artificial Intelligence), ML (Machine Learning), Blockchain (BC) and Deep Learning. Each of these techniques acts as a support for realising solutions that were not possible earlier. Real-time decision making, mission critical data analytics, predictive maintenance, natural language processing are some of the areas where the above-mentioned techniques play a significant role.

Blockchain is a disruptive technology which enables a platform for digital transformation of businesses[1]. It can equally benefit startups as well as well renowned enterprises for providing ledgerbased record keeping and secure transactions. It is an alternate computing model that provides improved security and reduced costs for transactions compared to traditional methods. It provides a mechanism for both human as well non-human entities to establish a trust based immutable transaction bridge for exchange based on values for a diverse assets range. One thing that needs to be understood is BC is still in its nascent stages and it is not under any legal, government body or traditional institutions. Its completely decentralised, distributed and highly secure.

So, what does the combination of BC and IoT brings to the table? Listed below are few of the advantages of combining these two cutting-edge technologies [2].

- $\quad$ Records created in BC are transparent which makes tracking and analysis of any activity possible by authorised network users. This is highly useful to track outages, failures, possible data leaks, identification of weak links and take the necessary countermeasures.

- $\quad$ Since all the records are encrypted, anyone involved in a transaction can trust the data and all transaction will be recorded by machines which avoids the human oversight.

- BC provides options for creating "smart contracts" that allows for executing agreements when specific conditions are met. This is highly useful in case of delivery-based services. 
- $\quad$ BC improves the overall security of the IoT network which primarily constitutes highly sensitive personal data.

Even though financial sector was the first targeted sector for realising the full potential of $\mathrm{BC}$, its is now found that it can be extended across multiple sectors that includes manufacturing, supply chain, healthcare, government, supply chain, education and energy [3]. The one major benefit of employing BC was the cost savings that could achieved for performing interoffice transactions and asset tracking. The remainder of the paper is organised into following sections. Section points out the various strengths and weaknesses of BC. Section 3 gives a brief idea of possible use cases of blockchain. Section 4 briefly summarises the opportunities and threats of BC, Section 5 depicts a real-time use case in India where $\mathrm{BC}$ has been used to achieve significant benefits. and finally, section 6 presents the concluding remarks and future research directions.

\section{Blockchain Strengths and weaknesses}

The various strengths of BC technology can be listed down as follows

- Distributed resilience and control

- Decentralized Network Architecture

- Fully Open Source

- Security and modern cryptography

- Asset tracking and maintenance

- Dynamic and fluid value exchange

The potential weaknesses exhibited by $\mathrm{BC}$ may include the following factors

- Lack of ledger interoperability

- Customer unfamiliarity and poor user experience

- Lack of governmental, tested technology

- Key management

- Skills scarcity and skill building costs

- Immature scalability

\section{Possible use cases of Blockchain}

Figure 1 depicts the possible scenarios in which Integration or adoption of $\mathrm{BC}$ technology is possible. In this section some existing IoT application domains are considered and how $\mathrm{BC}$ can help in each of these domains is briefly discussed.

\section{A. Healthcare}

In healthcare systems, medical scans and records are used to produce accurate diagnosis. Input to these systems is sensitive personal data. To meet expected standards fixed by some business deals, large amount of money should be used with respect to data security. Loss of personal data is increasingly high due to break in large scale data. Data that are present inside the Blockchain databases are in an encrypted state. All the private keys must be kept safe and the data range of private keys are of few kilobytes of data. Personal healthcare devices (PHD), Assisted Living (AL), wearable devices can all benefit by the security provided by $\mathrm{BC}$ technology.

\section{B. Manufacturing}

BC asset tracking capability can help in inventory management and predictive maintenance of the manufacturing domain [4]. Automated assembly lines can suffer from demanding workloads and $\mathrm{BC}$ technology can help in keeping track of wear and tear and general machine parts health and efficiently plan the predictive maintenance routines. The entire management from raw materials procurement, bill generation, spares maintenance, shipment delivery assist, order placements can all be monitored and tracked with the help of BC. Industrial IoT or Industry 4.0 can hugely benefit by integration of $\mathrm{BC}$.

\section{Agriculture}

$\mathrm{BC}$ can be used to manage the overall Agriculture process and help in achieving the goal of performing tasks with minimal human intervention. Periodic tracking of various stages of growth, precision agriculture practices, weather cycle management, soil nutrients and water requirements can all be processed using $\mathrm{BC}$ technology. The farmers can be provided with real-time monitoring updates about seed availability, pests control, irrigation and fertilizer requirements. $\mathrm{BC}$ can be used to provide convenience and improved efficiency by making use of IoT network as its backbone and cloud services for better analysis. Adopting better farming practises is the need of the hour.

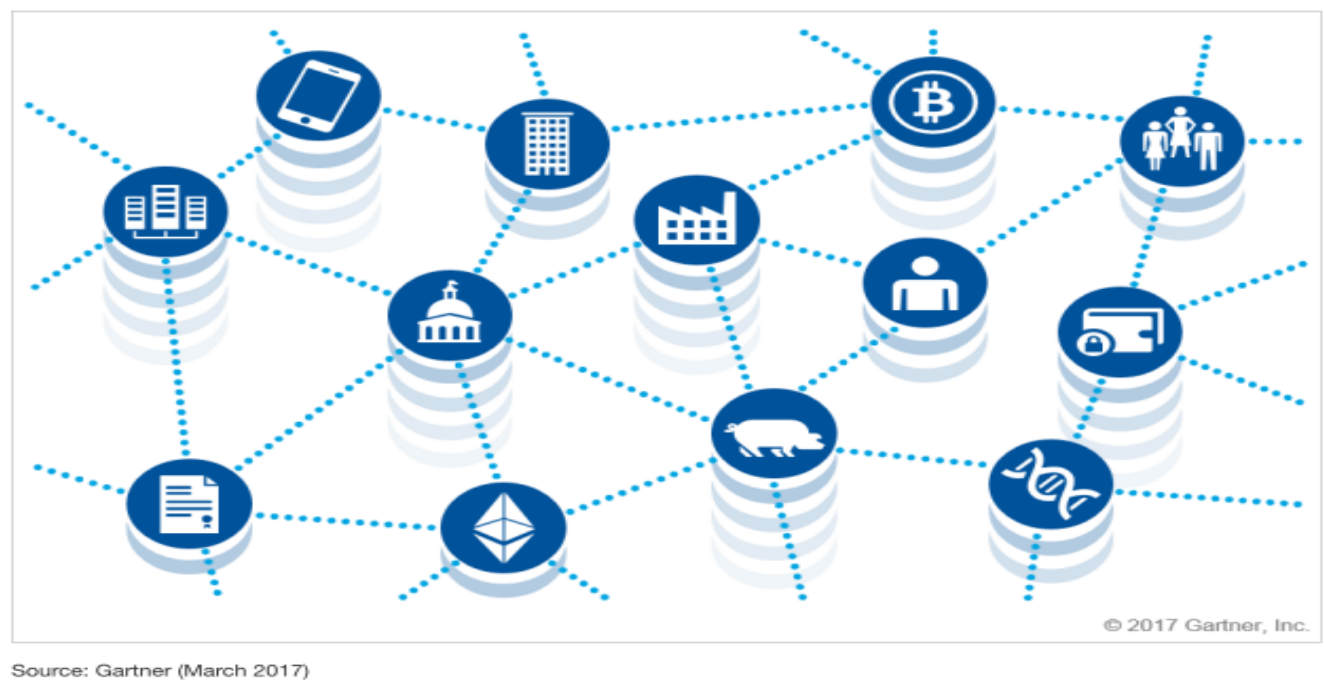

\section{Artificial Intelligence (AI)}

AI in simple terms can be defined process of building machines that require intelligence to accomplish tasks. AI helps in making informed decisions and choosing the best available option out of the solutions that are possible. Deep learning, machine learning and artificial neural networks are the techniques that help us to achieve the dream of AI. BC helps in creating a platform to 
understand, explain and track decision made by AI. Similarly, AI helps in understanding $\mathrm{BC}$ transactions better that what humans are capable of. Since BC records are in an encrypted state, securing private keys alone may be sufficient which typically consumes few kilobytes of data. When $\mathrm{BC}$ and $\mathrm{AI}$ are combined it makes people feel safer about adoption of AI to achieve transparency and understand the decision making intelligence of $\mathrm{AI}$ far better. The combination of $\mathrm{AI}$ and $\mathrm{BC}$ creates a platform on which the sensitive IoT data can be processed by smart machines in a secure fashion.

\section{E. Supply chains}

Networks owned by multiple organizations will have data transactions and records that can be tracked as data and pass between the supply chains. Blockchain records are transparent by nature and can be accessed by anyone connected to the network. If anything goes wrong, breakage occurs but Blockchain record makes it easier to identity the fault and take remedial action. Data involved in the supply chain can be trusted by all parties when encryption and distributed storage is used. Details of transactions are recorded securely by machines without human intervention. Without write access, no human will be allowed to overwrite Blockchain information with inaccurate information.
Many e-governance measures, documents validation, distribution of benefits, property registrations and sale, voting, budget discussions, currency exchange and remittance, tracking of public transport are some of the possible use cases where $\mathrm{BC}$ can play a major role [5]. One interesting and lucrative use case is creating new ventures and marketplaces where industry, angel investors, venture capitalists, research, developers and start-ups can all come together to pool in the resources and achieve specific objectives. Any application in which the records can be used but not modified can be implemented using BC technology.

\section{Blockchain Opportunities and Threats}

In this section we have illustrated the various opportunities that $\mathrm{BC}$ has created. It is pointed out earlier too that the fundamental utility for development of BC technology was financial management [6], [7]. When people got hold of what all are the possibilities that can be explored it opened a world of opportunities as depicted below in Figure 2.

The threats presented above are majorly due to the fact that still $\mathrm{BC}$ technology has not reached its full potential. When more and more adoption take place, we can expect the minimization of threats and better benefits can be achieved using BC.

\section{F. Governments}

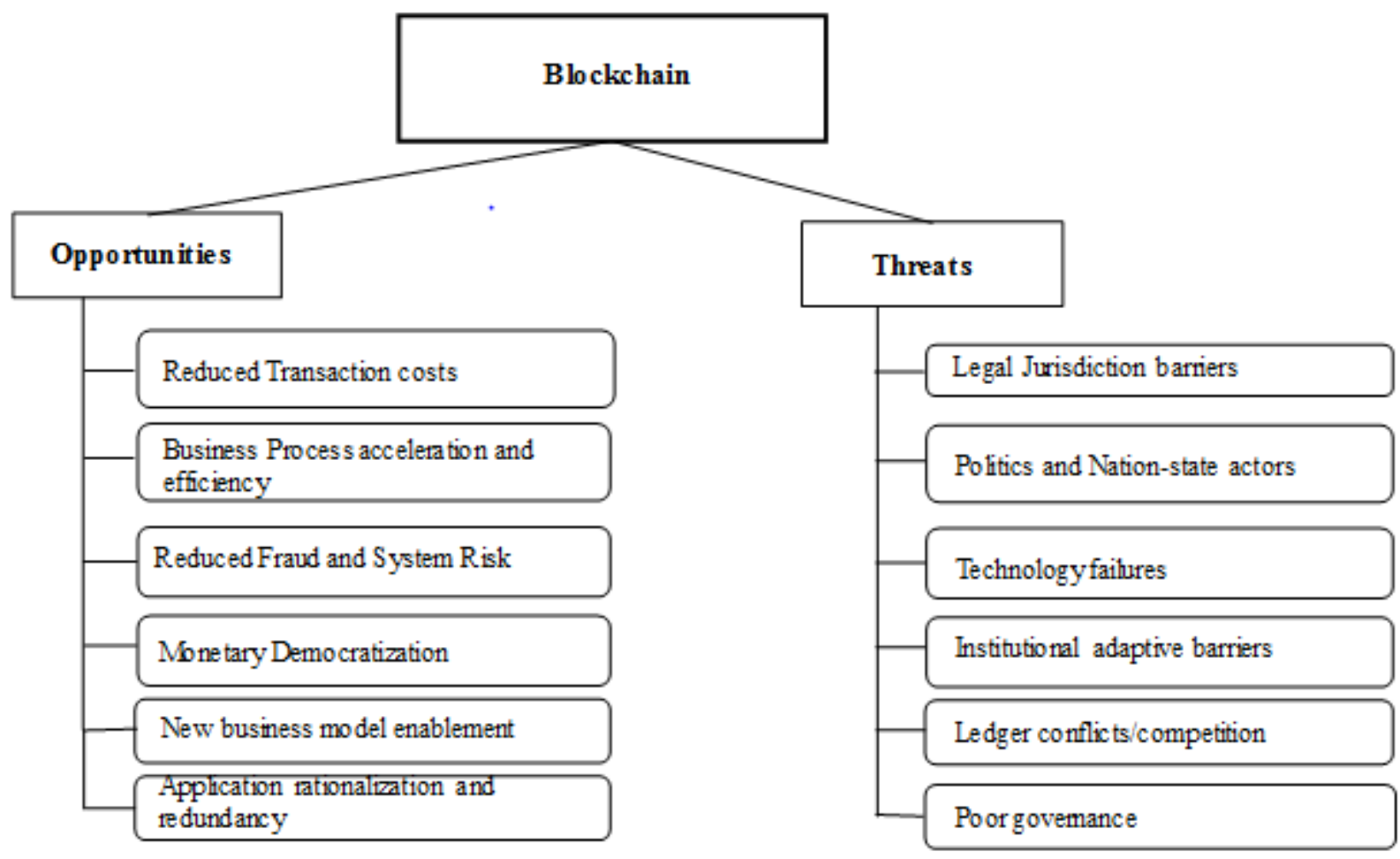

Fig. 2: Blockchain Opportunities and challenges

\section{Real-time Use case - Fintech Valley Vizag}

In 2016, the government of Andhra Pradesh, India initiated the "Fintech Valley" project in the port city of Vishakhapatnam (Vizag) which lead to establishment of an ecosystem that is at par with the global standards. It was joint venture of corporates, governments, academia, entrepreneurs and investors. By late 2017 ,

the project was able to attract investments close to 900 million US dollars and created job opportunities for more than 5000 people. Owing to the success of this venture the government of Andhra
Pradesh has started to use $\mathrm{BC}$ technology for maintaining land records and streamlining automobile registrations. The state has further partnered with private organisations from India ad abroad to procure records, data and maintenance data to achieve faster implementations of government measures and create tamper proof secure digital transactions which can help for streamlining the various operations of the government. The project has also opened its doors into the Banking finance and insurance industries to address common problems and come up with solutions. Other key areas where the focus lies include digital security for documents, finance and public transport. Fintech valley Vizag now acts as a beacon of opportunities with monetary benefits provided to bring 
in best available talents and develop better solutions to existing problems.

\section{Conclusion and Future Directions}

Blockchain's growth has opened up various avenues where better solutions are expected to be achieved. The BC technology is still immature and plunging head first into it without considering the limitations and threats may not work well for businesses and organisations. Blockchain provides an architecture in which applications, management of records and transactions can be rationalized, and removes the requirement of establishing trust between two different entities. This paper tries to bring up a picture of what currently BC is capable of and what are the possibilities, opportunities and challenges associated with adoption of this technology. There is every possibility that convergence of $\mathrm{BC}$ and IoT technologies becomes a absolute necessity in the near foreseeable future. The distributed nature of $\mathrm{BC}$ can help reduce the bottlenecks of existing IoT environment, improving the overall QoS of the network.

Future directions for taking forward the $\mathrm{BC}$ technology is to weigh down the fact that both IoT and BC are enthusiastically adopted by industry and the academia and it leads to widespread speculation of where the collaboration will lead to. BC technology is still in its nascent stages and people haven't got the full grasp of it yet. Risk assessment, Standardisation, common reference architecture, scalability are some of the future research directions to consider.

\section{References}

[1] D. Furlonger and R. Valdes, "Practical Blockchain: A Gartner Trend Insight Report," Gart. Res., vol. G00325933, no. March, p. 3, 2017.

[2] "The CIO's Guide to Blockchain - Smarter with Gartner", www.gartner.com/smarterwithgartner/the-cios-guide-toblockchain/, Date accessed 2018-03-22.

[3] J. Heng, R. Kandaswamy, N. Barton, and D. Groombridge, "Market Guide for Blockchain Consulting and Proof-of-Concept Development Services," Gart. Inc., no. February, 2017.

[4] J. J. Sikorski, J. Haughton, and M. Kraft, "Blockchain technology in the chemical industry: Machine-to-machine electricity market,' Appl. Energy, vol. 195, pp. 234-246, 2017.

[5] IBM, "IoT Lab Services blockchain offerings - Fast-track your IoT and blockchain project today with IoT Lab Services," 2016.

[6] A. Dorri, S. S. Kanhere, and R. Jurdak, "Blockchain in internet of things: Challenges and Solutions," 2016.

[7] M. Banerjee, J. Lee, and K.-K. R. Choo, "A blockchain future to Internet of Things security: A position paper," Digit. Commun. Networks, 2017.

[8] S.V.Manikanthan and V.Rama"Optimal Performance of Key Predistribution Protocol In Wireless Sensor Networks" International Innovative Research Journal of Engineering and Technology ,ISSN NO: 2456-1983,Vol-2,Issue -Special -March 2017.

[9] T. Padmapriya and V. Saminadan, "Priority based fair resource allocation and Admission Control Technique for Multi-user Multiclass downlink Traffic in LTE-Advanced Networks", International Journal of Advanced Research, vol.5, no.1, pp.1633-1641, January 2017. 